\title{
Clinical course and characteristics of Corona Virus Disease 2019 in Xiaoshan, Hangzhou: A Retrospective study
}

\section{Yongtao Zheng}

Department of Infectious Disease, Xiaoshan Center for Disease control and Prevention and Institute of Clinical Epidemiology and Evidence-based medicine, Tongji University School of Medicine Hexiang Jia

Department of Infectious Disease, Xiaoshan Center for Disease control and Prevention

\section{Fuliang Wang}

Department of Infectious Disease, Xiaoshan Center for Disease control and Prevention

\section{Xiangjue Sun}

Department of Infectious Disease, Xiaoshan Center for Disease control and Prevention

\section{Dongbo Yang}

Department of Public Health, Community Healthcare Center of Guali Town, Hangzhou

\section{Miaojian Xu}

Department of Infectious Disease, Xiaoshan Center for Disease control and Prevention

\section{Diwei Xu}

Department of Infectious Disease, Xiaoshan Center for Disease control and Prevention

\section{Chao Lu}

Department of Infectious Disease, Xiaoshan Center for Disease control and Prevention

\section{Bo Ge}

Department of Infectious Disease, Xiaoshan Center for Disease control and Prevention

\section{Shenglan Chen}

Department of Infectious Disease, Xiaoshan Center for Disease control and Prevention

\section{Biao Li}

Department of Infectious Disease, Xiaoshan Center for Disease control and Prevention

\section{Ting Chen}

Department of Infectious Disease, Xiaoshan Center for Disease control and Prevention

\section{Zhibo Li}

Department of Infectious Disease, Xiaoshan Center for Disease control and Prevention

\section{Yuanmei Feng}

Department of Infectious Disease, Xiaoshan Center for Disease control and Prevention

\section{Fenhua Lai ( 928497368@qq.com )}

Department of Infectious Disease, Xiaoshan Center for Disease control and Prevention 


\section{Research Article}

Keywords: COVID-19, SARS-Cov-2, Epidemiological, Clinical, Retrospective

Posted Date: April 9th, 2020

DOI: https://doi.org/10.21203/rs.3.rs-22080/v1

License: (a) (i) This work is licensed under a Creative Commons Attribution 4.0 International License. Read Full License 


\section{Abstract}

Background: The World Health Organization declared the outbreak a public health emergency of international concern (Corona Virus Disease 2019) on January 30th, this study aimed to investigate the epidemiological and clinical characteristics of Corona Virus Disease 2019 in Xiaoshan, Hangzhou, and evaluate scientific basis for disease control and prevention.

Methods: A total of 30 patients had been admitted to hospital from Jan 22 to Feb 22, 2020, all of them were laboratory confirmed SARS-Cov-2. Demographic, epidemiological, clinical, laboratory data were collected from Hospital information system and Epidemiological investigation reports. All data was performed by descriptive analysis, Chi-square test or non-parametric Mann-Whitney $\mathrm{U}$ test, as appropriate. Two sided $p$ value less than 0.05 was considered statistically significant.

Results: 30 patients were enrolled, the median age was 44.5 years (IQR 33.8-52.3) and $17(56.7 \%)$ patients were female, $14(46.7 \%)$ patients were native and had no exposure to Hubei Province. At the time of study submission, only one patient had not been discharged and no patients died during the study. The median hospital stay was 16.0 days (IQR 12.5-20.5) and the median course of disease was 20.5 days (IQR 17.023.3). The most common symptoms were fever (66.7\%), dry cough $(26.7 \%)$, and pharyngalgia $(23.3 \%)$ on first admission. Most patients were generally illness or more mild, but $10(33.3 \%)$ patients received oxygen therapy and $14(46.7 \%)$ patients received hormone therapy during their hospitalization. Almost half of patients showed mild lymphocytopenia and $40 \%$ patients had elevated concentrations of CRP in the early stages of COVID-19.

Conclusions: Among the 30 patients were confirmed with SARA-Cov-2 infection in Xiaoshan, Hangzhou, most of them had clinical presentation of respiratory tract infection, but the median course of disease was more than 2 weeks. Further systematic prospective studies about COVID-19 should be urgently needed.

\section{Background}

In December 2019, an unknown cause pneumonia occurred and now known as Severe acute respiratory syndrome coronavirus 2 (SARA-Cov-2) infection in Wuhan, Hubei

Province, China [1-4]. The World Health Organization (WHO) declared the outbreak a public health emergency of international concern on January 30th[5].On February 11th, 2020, the novel coronavirus pneumonia was named Corona Virus Disease 2019 (COVID-19) by WHO. COVID-19 had rapidly spread from Wuhan to other areas, by 5th March, 2020, 80565 confirmed cases and 3015 deaths had been reported in China, and 14768 cases were reported in 85 countries and 6 continents[6]. The SARA-Cov-2 was the seventh member of enveloped RNA coronavirus and Full-genome sequencing indicated that SARACov-2 was a distinct clade from the beta coronaviruses associated with human severe acute respiratory syndrome (SARS) and Middle East respiratory syndrome (MERS)[7]. Previous a study suggested that SARACov-2 spread to humans occurred via transmission from wild animals illegally sold in a local Seafood market, Wuhan[8].The disease causes clinical symptoms in patients including fever, dry cough, productive 
cough, dyspnea, myalgia, fatigue, normal or decreased leukocyte counts, and radiographic evidence of pneumonia[9], it could even cause patient death in severe cases. In this study, we retrospective reviewed 30 patients with laboratory confirmed SARS-Cov-2 infections in Xiaoshan, and epidemiological and clinical characteristics had been collected. Thus to provide scientific basis for prevention and control of COVID-19 spreads at grassroots units.

\section{Methods}

\section{Design and participants}

A retrospective and observational study enrolled all patients infected with the SARA-Cov-2 in Xiaoshan from January 22 to February 11, 2020. A total of 30 patients were admitted to The First People's Hospital of Xiaoshan, Hangzhou Sixth People's Hospital and

The First Hospital of Zhejiang Province. The protocol for this study was approved by the Ethics Committee of Xiaoshan Center for Disease control and Prevention. All patients in study who were diagnosed as infecting SARA-Cov-2 according to WHO guidance[10], and informed consent was obtained for this study.

\section{Data collection}

Hospital information system and Epidemiological investigation reports were used to collect patients' health-related information including demographic, epidemiological, clinical, laboratory data. All data were reviewed by a trained team of public health physicians. Demographic data were recorded including gender, age, weight, height, occupation, permanent residence, family relationship. Epidemiological data was reported including onset date, family clustering (Great than or equal to one infected family member residing with the patient), exposure and linkage to Hubei Province (close contact with individuals from Hubei Province less than or equal to two weeks before the onset of infection). Clinical data including admission date, discharge date, body temperature, systolic blood pressure (SBP) and diastolic blood pressure (DBP), clinical severity, clinical symptoms, Chest Computed Tomography report (CT report), chronic disease, oxygen therapy, hormone therapy. Meanwhile, clinical severity was categorized into asymptomatic infection, mild illness, generally illness, and severe illness, critical illness[11]. Clinical symptoms were recorded including fever, shiver, dry cough, expectoration, nasal congestion, runny nose, pharyngalgia, headache and dizziness, fatigue, myalgia, dyspnea, vomiting, diarrhea. Chest CT report was measured with four response categories including without lung CT abnormalities, left lung ground glass opacity, right lung ground glass opacity, and bilateral ground glass opacity. And chronic disease was summarized as hypertension, diabetes, cardiovascular disease, chronic obstructive pulmonary disease (COPD), chronic kidney disease, chronic liver disease. Laboratory data including white blood cell count, lymphocyte count, lymphocyte ratio, neutrophil count, neutrophil ratio, C-reactive protein (CRP), hemoglobin, platelet count. Body Mass Index (BMI) was calculated as weight in kilograms divided by height in meters squared $\left(\mathrm{kg} / \mathrm{m}^{2}\right)$. Hospital stay was calculated in days by subtracting the discharge date and admission date (days), and Course of disease was calculated as the discharge date minus the onset date (days). 


\section{Detection method for SARS-Cov-2}

Throat swab or nasal swab samples were collected from all of patients after admission, and tested for SARS-Cov-2 with real-time RT-PCR Kit which was recommended by the Chinese Center for Disease Control and Prevention. All samples were tested by Xiaoshan Center for Disease control and Prevention according to the relevant guidelines of the World Health Organization[12].The threshold value (Ct value) greater than or equal to 40 was defined as a negative result, and $\mathrm{Ct}$ value less than 37 was defined as a positive result. If the Ct value was between 37 and 40, it was recommended to repeat the real-time RT-PCR, if the redo result less than 40 , and the amplification curve had obvious peaks, then the result was defined as positive, otherwise it was negative. Cases of SARS-Cov-2 infection were defined as throat swab or nasal swab samples with the positive test result from Xiaoshan Center for Disease control and Prevention.

\section{Criteria of discharged from hospital}

Base on the COVID-19 treatment protocol (Sixth Edition)[11], Temperature recovered for more than 3 days; Respiratory symptoms improved; Chest CT report shows marked improvement in acute exudative lesions; Have twice negative nucleic acid test of respiratory samples (at least one day apart). Meet the above all conditions that can be discharged.

\section{Statistical analysis}

EpiData 3.1 (Odense, Denmark) was used for data input, and statistical management and analyses were performed using SAS Version 9.4 (SAS Institute, Cary, NC). Summary statistics for continuous variables were expressed as median and interquartile range (IQR), which were compared using the non-parametric Mann-Whitney $\mathrm{U}$ test. For categorical variables, we used frequency rates and percentages, the Chi-squared test was used to analyze the differences, and in addition, Fisher exact test was used when the data were limited. Two-sided $p$ value less than 0.05 was considered statistically significant.

\section{Results}

This study participants included 30 hospitalized patients with confirmed COVID-19 in Xiaoshan, Hangzhou, and as of February 27, 29 patients had been cured and discharged, and 1 patient was still in hospital without death. The median age was 44.5 years (IQR $33.8-52.3$ ), the median BMI was $24.3 \mathrm{~kg} / \mathrm{m}^{2}$ (IQR 20.7-26.0) and the majority of patients were female (56.7\%). Demographic and epidemiological characteristics of study participants by gender were summarized in Table 1 . With respect to occupation, Employees accounted for $40.0 \%$ of all patients, followed by self-employed (23.3\%), retirees $(20.0 \%)$, agricultural worker (10.0\%), and students (6.7\%), and none of the them were medical staff. $14(46.7 \%)$ patients were native and showed to be neither visiting in Hubei Province nor having family members who living in Hubei Province, the rest of them had direct or indirect linkage to Hubei Province. In addition, this 
study had 16 family clustering cases. There were no significant differences between male and female in age, occupation, exposure to Hubei Province, and family clustering cases.

Table 1 Demographic and epidemiological characteristics of 30 patients by gender

\begin{tabular}{|c|c|c|c|c|}
\hline & Total $(\mathrm{n}=30)$ & Male $(n=13)$ & Female $(n=17)$ & $p$ value \\
\hline Age (year) & $\begin{array}{c}44.5(33.8- \\
52.3)\end{array}$ & $44(33.0-53.0)$ & $45(34.0-52.0)$ & 0.869 \\
\hline BMI $\left(\mathrm{kg} / \mathrm{m}^{2}\right)$ & $\begin{array}{c}24.3(20.7- \\
26.0)\end{array}$ & $\begin{array}{c}25.1(21.5- \\
27.6)\end{array}$ & $\begin{array}{c}22.6(20.0- \\
25.1)\end{array}$ & 0.113 \\
\hline Occupation & & & & 0.210 \\
\hline Employee & $12(40.0 \%)$ & $8(61.5 \%)$ & $4(23.5 \%)$ & \\
\hline Self-employed & $7(23.3 \%)$ & $2(15.4 \%)$ & $5(29.4 \%)$ & \\
\hline Retiree & $6(20.0 \%)$ & $2(15.4 \%)$ & $4(23.5 \%)$ & \\
\hline Agricultural worker & $3(10.0 \%)$ & $0(0.0 \%)$ & $3(17.6 \%)$ & \\
\hline Student & $2(6.7 \%)$ & $1(7.7 \%)$ & $1(5.9 \%)$ & \\
\hline $\begin{array}{l}\text { Exposure to Hubei } \\
\text { Province }\end{array}$ & $14(46.7 \%)$ & $4(30.8 \%)$ & $10(58.8 \%)$ & 0.127 \\
\hline Family clustering & $16(53.3 \%)$ & $6(46.2 \%)$ & $10(58.8 \%)$ & 0.475 \\
\hline
\end{tabular}

The clinical characteristics of 30 patients by gender were shown in Table 2 . Of the 30 patients, Body temperature, blood pressure, Chest CT were monitored on hospital admission, the median body temperature, SBP, DBP were $37.9^{\circ} \mathrm{C}$ (IQR 36.9-38.2), $130.0 \mathrm{mmHg}$ (IQR $\left.120.0-136.0\right), 80.0 \mathrm{mmHg}$ (IQR 70.0-88.0), respectively. Chest CT reported that most patients (70.0\%) had bilateral ground glass opacity, which indicated that had inflammation in both lungs. 29 patients had been cured and discharged and the median hospital stay was 16.0 days (IQR 12.5-20.5), the median course of disease was 20.5 days (IQR 17.0-23.3). Furthermore, 10 (33.3\%) patients received oxygen therapy and 14 (46.7\%) patients received hormone therapy during their hospitalization. Generally illness or more mild illness was the most clinical severity in this study, accounting for more than $60 \%$, meanwhile, it was worth mentioning that there were 3 patients confirmed asymptomatic infection, which showed that only SARS-Cov-2 tested was positive. The most common symptoms were fever (66.7\%), dry cough (26.7\%), and pharyngalgia (23.3\%), all of them had no dyspnea, vomiting, diarrhea.

Only a few patients had chronic disease including hypertension (10\%), diabetes (10\%), cardiovascular disease (3.3\%) and COPD (3.3\%).

Table 2 Clinical characteristics of 30 patients by gender 


\begin{tabular}{|c|c|c|c|c|}
\hline & Total $(n=30)$ & Male $(n=13)$ & Female $(\mathrm{n}=17)$ & $p$ value \\
\hline Body temperature $\left({ }^{\circ} \mathrm{C}\right)$ & $37.9(36.9-38.2)$ & $37.9(37.6-38.4)$ & $37.8(36.8-38.2)$ & 0.408 \\
\hline $\mathrm{SBP}(\mathrm{mmHg})$ & $\begin{array}{c}130.0(120.0- \\
136.0)\end{array}$ & $\begin{array}{c}133.0(130.0- \\
140.0)\end{array}$ & $\begin{array}{c}123.0(110.0- \\
132.5)\end{array}$ & 0.009 \\
\hline DBP (mmHg) & $80.0(70.0-88.0)$ & $88.0(85.0-90.0)$ & $78.0(64.5-80.0)$ & $<0.001$ \\
\hline Hospital stay (days) & $16.0(12.5-20.5)$ & $17.5(12.3-19.5)$ & $16.0(12.0-22.0)$ & 0.965 \\
\hline Course of disease (days) & $20.5(17.0-23.3)$ & $20.5(15.5-22.5)$ & $20.0(17.0-25.5)$ & 0.494 \\
\hline Clinical severity & & & & 0.282 \\
\hline Asymptomatic infection & $3(10.0 \%)$ & $0(0.0 \%)$ & $3(17.6 \%)$ & \\
\hline Mild illness & $6(20.0 \%)$ & $3(23.1 \%)$ & $3(17.6 \%)$ & \\
\hline Generally illness & $18(60.0 \%)$ & $8(61.5 \%)$ & $10(58.8 \%)$ & \\
\hline Severe illness & $1(3.3 \%)$ & $0(0.0 \%)$ & $1(5.9 \%)$ & \\
\hline Critical illness & $2(6.7 \%)$ & $2(15.4 \%)$ & $0(0.0 \%)$ & \\
\hline \multicolumn{5}{|l|}{ Clinical symptoms } \\
\hline Fever & $20(66.7 \%)$ & $10(76.9 \%)$ & $10(58.8 \%)$ & 0.515 \\
\hline Shiver & $1(3.3 \%)$ & $1(7.7 \%)$ & $0(0.0 \%)$ & 0.433 \\
\hline Dry cough & $8(26.7 \%)$ & $3(23.1 \%)$ & $5(29.4 \%)$ & 1.000 \\
\hline Expectoration & $3(10.0 \%)$ & $2(15.4 \%)$ & $1(5.9 \%)$ & 0.565 \\
\hline Nasal congestion & $3(10.0 \%)$ & $1(7.7 \%)$ & $2(11.8 \%)$ & 1.000 \\
\hline Runny nose & $2(6.7 \%)$ & $1(7.7 \%)$ & $1(5.9 \%)$ & 1.000 \\
\hline Pharyngalgia & $7(23.3 \%)$ & $4(30.8 \%)$ & $3(17.6 \%)$ & 0.666 \\
\hline Headache and Dizziness & $3(10.0 \%)$ & $2(15.4 \%)$ & $1(5.9 \%)$ & 0.565 \\
\hline Fatigue & $4(13.3 \%)$ & $4(30.8 \%)$ & $0(0.0 \%)$ & 0.026 \\
\hline Myalgia & $1(3.3 \%)$ & $0(0.0 \%)$ & $1(5.9 \%)$ & 1.000 \\
\hline Dyspnea & $0(0.0 \%)$ & $0(0.0 \%)$ & $0(0.0 \%)$ & - \\
\hline Vomiting & $0(0.0 \%)$ & $0(0.0 \%)$ & $0(0.0 \%)$ & - \\
\hline Diarrhea & $0(0.0 \%)$ & $0(0.0 \%)$ & $0(0.0 \%)$ & - \\
\hline Chest CT report & & & & 0.387 \\
\hline $\begin{array}{r}\text { Without lung CT } \\
\text { abnormalities }\end{array}$ & $4(13.3 \%)$ & $1(7.7 \%)$ & $3(17.6 \%)$ & \\
\hline $\begin{array}{r}\text { Left lung ground glass } \\
\text { opacity }\end{array}$ & $2(6.7 \%)$ & $1(7.7 \%)$ & $1(5.9 \%)$ & \\
\hline $\begin{array}{r}\text { Right lung ground glass } \\
\text { opacity }\end{array}$ & $3(10.0 \%)$ & $0(0.0 \%)$ & $3(17.6 \%)$ & \\
\hline $\begin{array}{r}\text { Bilateral ground glass } \\
\text { opacity }\end{array}$ & $21(70.0 \%)$ & $11(84.6 \%)$ & $10(58.8 \%)$ & \\
\hline \multicolumn{5}{|l|}{ Chronic disease } \\
\hline Hypertension & $3(10.0 \%)$ & $3(23.1 \%)$ & $0(0.0 \%)$ & 0.070 \\
\hline Diabetes & $3(10.0 \%)$ & $2(15.4 \%)$ & $1(5.9 \%)$ & 0.565 \\
\hline Cardiovascular disease & $1(3.3 \%)$ & $1(7.7 \%)$ & $0(0.0 \%)$ & 0.433 \\
\hline COPD & $1(3.3 \%)$ & $0(0.0 \%)$ & $1(5.9 \%)$ & 1.000 \\
\hline Chronic kidney disease & $0(0.0 \%)$ & $0(0.0 \%)$ & $0(0.0 \%)$ & - \\
\hline Chronic liver disease & $0(0.0 \%)$ & $0(0.0 \%)$ & $0(0.0 \%)$ & - \\
\hline Oxygen therapy & $10(33.3 \%)$ & $5(38.5 \%)$ & $5(29.4 \%)$ & 0.896 \\
\hline
\end{tabular}

\begin{tabular}{lllll}
\hline Hormone therapy & $14(46.7 \%)$ & $6(46.2 \%)$ & $8(47.1 \%)$ & 0.961 \\
\hline
\end{tabular}


Laboratory findings on first admission were shown in Table 3. The levels of white blood cell count decreased in 4 (13.3\%) patients and increased in 1 (3.3\%) patient. Lymphocyte count was above the normal range in $1(3.3 \%)$ patients and below the normal range in $12(40.0 \%)$ patients. $10(33.3 \%)$ patients had lymphocyte ratio below the normal range. The neutrophil count of $2(6.7 \%)$ showed neutrophilia and 7 $(23.3 \%)$ showed neutropenia, neutrophil ratio increased in $5(16.7 \%)$ patients. 12 (40\%) patients had CRP levels above the normal range. $9(30 \%)$ patients had hemoglobin decreased and all of whom were female. In addition, there were only 1 (3.3\%) patient had platelet count increased and decreased, respectively.

Table 3 Laboratory characteristics of 30 patients by gender

\begin{tabular}{|c|c|c|c|c|c|}
\hline & $\begin{array}{c}\text { Normal } \\
\text { range }\end{array}$ & Total $(n=30)$ & Male $(n=13)$ & Female $(\mathrm{n}=17)$ & $p$ value \\
\hline $\begin{array}{l}\text { White blood cell count } \\
\left(\times 10^{9} / \mathrm{L}\right)\end{array}$ & $3.5-9.5$ & $4.9(3.8-6.1)$ & $5.4(4.1-8.0)$ & $4.5(3.8-5.9)$ & 0.229 \\
\hline Increased & & $1(3.3 \%)$ & $1(7.7 \%)$ & $0(0.0 \%)$ & 0.439 \\
\hline Decreased & & $4(13.3 \%)$ & $1(7.7 \%)$ & $3(17.6 \%)$ & \\
\hline $\begin{array}{l}\text { Lymphocyte count } \\
\left(\times 10^{9} / \mathrm{L}\right)\end{array}$ & $1.1-3.2$ & $1.2(0.8-1.8)$ & $1.2(0.8-1.6)$ & $1.3(0.8-1.9)$ & 0.742 \\
\hline Increased & & $1(3.3 \%)$ & $0(0.0 \%)$ & $1(5.9 \%)$ & 0.561 \\
\hline Decreased & & $12(40.0 \%)$ & $4(30.8 \%)$ & $8(47.1 \%)$ & \\
\hline Lymphocyte ratio (\%) & $20.0-50.0$ & $26.4(16.7-35.7)$ & $20.9(15.5-31.9)$ & $32.9(22.3-35.9)$ & 0.170 \\
\hline Increased & & $0(0.0 \%)$ & $0(0.0 \%)$ & $0(0.0 \%)$ & 0.362 \\
\hline Decreased & & $10(33.3 \%)$ & $6(46.2 \%)$ & $4(23.5 \%)$ & \\
\hline $\begin{array}{l}\text { Neutrophil count } \\
\left(\times 10^{9} / \mathrm{L}\right)\end{array}$ & $1.8-6.3$ & $3.25(1.96-3.69)$ & $3.6(2.0-4.7)$ & $2.9(1.8-3.4)$ & 0.300 \\
\hline Increased & & $2(6.7 \%)$ & $2(15.4 \%)$ & $0(0.0 \%)$ & 0.335 \\
\hline Decreased & & $7(23.3 \%)$ & $3(23.1 \%)$ & $4(223.5 \%)$ & \\
\hline Neutrophil ratio (\%) & $40.0-75.0$ & $64.1(53.5-69.2)$ & $67.2(56.1-77.6)$ & $58.1(53.4-65.0)$ & 0.059 \\
\hline Increased & & $5(16.7 \%)$ & $4(30.8 \%)$ & $1(5.9 \%)$ & 0.138 \\
\hline Decreased & & $0(0.0 \%)$ & $0(0.0 \%)$ & $0(0.0 \%)$ & \\
\hline $\mathrm{CRP}(\mathrm{mg} / \mathrm{L})$ & $0.0-10.0$ & $8.3(2.1-24.2)$ & $15.7(6.3-33.5)$ & $5.7(1.2-14.0)$ & 0.059 \\
\hline Increased & & $12(40.0 \%)$ & 7 (53.8\%) & $5(29.4 \%)$ & 0.176 \\
\hline Hemoglobin $(\mathrm{g} / \mathrm{L})$ & $\begin{array}{l}130.0- \\
175.0\end{array}$ & $\begin{array}{c}137.0(127.0- \\
151.0)\end{array}$ & $\begin{array}{c}151.0(139.5- \\
159.0)\end{array}$ & $\begin{array}{c}127.0(124.5- \\
139.0)\end{array}$ & $<0.001$ \\
\hline Increased & & $0(0.0 \%)$ & $0(0.0 \%)$ & $0(0.0 \%)$ & 0.006 \\
\hline Decreased & & $9(30.0 \%)$ & $0(0.0 \%)$ & $9(52.9 \%)$ & \\
\hline $\begin{array}{l}\text { Platelet count } \\
\left(\times 10^{9} / \mathrm{L}\right)\end{array}$ & $\begin{array}{l}125.0- \\
350.0\end{array}$ & $\begin{array}{c}190.5(161.8- \\
239.5)\end{array}$ & $\begin{array}{l}196.0(164.5- \\
242.0)\end{array}$ & $\begin{array}{c}176.0(157.0- \\
246.0)\end{array}$ & 0.432 \\
\hline Increased & & $1(3.3 \%)$ & $1(7.7 \%)$ & $0(0.0 \%)$ & 0.179 \\
\hline
\end{tabular}

\begin{tabular}{llll} 
Decreased & $1(3.3 \%)$ & $1(7.7 \%)$ & $0(0.0 \%)$ \\
\hline
\end{tabular}

\section{Discussion}

In late January, 2020, the number of COVID-19 patients increased rapidly from Wuhan, Hubei Province to all over the world and had become a global health concern [4, 7]. 6 human coronaviruses including HCovOC43, HCoV-229E, HCoV-NL-63, HCoV-HKU1, SARS-CoV, and MERS-CoV were identified[13], SARS-CoV and MERS-CoV could cause severe respiratory syndrome in humans. The sequence of SARS-CoV-2 was 
relatively different from the six other coronavirus subtypes but can be classified as beta coronavirus[4].The recent studies reported rapid person-to-person transmission of SARS-Cov-2 had occurred[14, 15], and another study showed that the spread of SARS-Cov-2 may be stronger than that of SARS-CoV and MERSCoV[16], according to a previous study[15], the basic reproductive number $\left(R_{0}\right)$ was 2.2, which estimated that each patients could spread infection to 2.2 other people on average. The SARS-Cov- 2 was spread by close contact and respiratory droplet infection and had an incubation period of 3-7 days, the longest was not more than 14 days[17].

Xiaoshan is one of the districts of Hangzhou, Zhejiang Province, Chinese government announced a travel quarantine of Wuhan, Hubei Province, on January 23, 2020, because of the Spring Festival that Chinese had made several billion trips throughout China to celebrate the Lunar New Year[18]. This social phenomenon was considered a reason of COVID-19 increased spread of infection. Until now, the demographic, epidemiological, clinical and laboratory data of patients were insufficient, especially outside Hubei Province. By February 3rd, 2020, a total of 30 patients were diagnosed and enrolled in this retrospective study, to provide an insight into the control and prevention of COVID-19. The findings showed that $56.7 \%$ patients were female and the median age was 44.5 years. Based on previous studies, the age distribution of patients were mostly between $30-60$ years $[19,20]$, which was supported by this study. Moreover, other studies suggested that male patients might be the more susceptible to SARS-CoV-2 infection $[8,19]$, and the sample size may be insufficient to find significant differences between genders in this study. According to epidemiological data, only $46.7 \%$ patients had exposure to Hubei Province, additionally, 53.3\% patients were family clustering, that might demonstrate person-to-person transmission. This result was consistent with those of previous studies[14, 15,20], further studies should be needed to identify SARS-Cov-2 transmission and design interventions for these settings.

In terms of clinical characteristics, this study found that the median body temperature was $37.9^{\circ} \mathrm{C}$, and more than half $(66.7 \%)$ of the patients had fever, a portion of them had dry cough and pharyngalgia, resembling that in previous studies[8,20]. Moreover, 1 patient had atypical symptom (myalgia), and this study showed that 3 patients were asymptomatic infection, it may suggest that an even greater number of patients were overlooked because they may present with atypical symptoms of COVID-19. Of the 30 patients, 29 had been cured and discharged and the remaining one would be discharged soon, none of them were dead, one of the reasons was that most of them were mild and generally illness, another reason was that only a small portion of patients had chronic underlying diseases, such as hypertension, diabetes. As previously reported, patients with a history of chronic underlying diseases were at increased risk of becoming critical illness or death $[8,14]$. However, nearly half of the patients received hormone therapy, and a third received oxygen therapy, no specific treatment had been identified for coronavirus infection except for supportive care at this time[21], all patients were treated in isolation and their close contacts were quarantined, but the present study indicated the median hospital stay and the median course of disease were still more than two weeks, which would result in a serious waste of medical resources, cause considerable economic burden to patients or government. Accordingly, prevention and early diagnosis of this disease are very important. Additionally, Laboratory data were detected and almost half of patients showed mild lymphocytopenia in the early stages of COVID-19.40\% patients had elevated concentrations 
of CRP. These findings were similar to the previous studies [8,22]. One third of patients appeared to have hemoglobin decreased and all of were female, this result could be further confirmed through related studies.

There were several limitations to this study that should be noted. First, A retrospective and observational study may not fully assess the COVID-19 especially temporality, and thus the causal relationship was still indeterminate. A longitudinal follow-up study should be needed to address the limitation. Second, the sample size of raw data was insufficient and may not provide adequate statistical power to detect significant difference, especially for categorical variables with rare events, so not much emphasis was laid on presenting statistical evaluation. Third, the incubation period of COVID-19 was still not very clear in this study, and further follow-up would be needed for long-term results, so it was difficult to assess continued observations of the natural history of the disease. Moreover, some specific data from hospital was missing, such as total medical expenses, because the medical insurance system had not yet achieved the unified settlement within the medical alliance, therefore, health economics measures could not be collected for an economic evaluation.

\section{Conclusions}

Among the 30patients were confirmed with SARA-Cov-2 infection in Xiaoshan, Hangzhou, most of them had clinical presentation of respiratory tract infection, but the median course of disease was more than 2 weeks. Further systematic prospective studies about COVID-19 should be urgently needed.

\section{List Of Abbreviations}

SARA-Cov-2: Severe acute respiratory syndrome coronavirus 2

WHO: World Health Organization

COVID-19: Corona Virus Disease 2019

SARS: severe acute respiratory syndrome

MERS: Middle East respiratory syndrome

SBP: systolic blood pressure

DBP: diastolic blood pressure

CT: Computed Tomography

COPD: chronic obstructive pulmonary disease

CRP: C-reactive protein

BMI: Body Mass Index 
IQR: interquartile range

\section{Declarations}

\section{Ethics approval and consent to participate}

The protocol for this study was approved by the Ethics Committee of Xiaoshan Center for Disease control and Prevention. Informed consent was obtained from all patients.

\section{Consent for publication}

Not applicable.

\section{Availability of data and materials}

The datasets used and analyzed during the current study are available from the corresponding author on reasonable request.

\section{Competing interests}

The authors declare that they have no competing interests.

\section{Funding}

This study did not receive any specific grant from funding agencies in the public, commercial, or not-forprofit sectors.

\section{Authors' Contributions}

Yongtao Zheng and Hexiang Jia designed the research; Fenhua Lai, Miaojian Xu, Xiangjue Sun, Bo Ge, Diwei Xu collected the demographic and epidemiological data; Fuliang Wang, Dongbo Yang, Chao Lu, Shenglan Chen collected the clinical and laboratory data; Yongtao Zheng summarised all data; Yongtao Zheng and Biao Li conducted the data analyses; Yongtao Zheng wrote the draft of initial manuscript; Hexiang Jia and Fenhua Lai critically contributed to data reanalysis and draft revision; Ting Chen, Zhibo Li, Yuanmei Feng contributed to the interpretation of the data and preparation of the manuscript. Yongtao Zheng and Fenhua Lai contributed to the work equally and could be regarded as co-first authors.

\section{Acknowledgements}

We thanks to all patients and their families involved in the study. 


\section{References}

1. Lu H, Stratton C, Tang Y-W: Outbreak of Pneumonia of Unknown Etiology in Wuhan China: the Mystery and the Miracle. Journal of Medical Virology 2020, 92.

2. Hui DS, I Azhar E, Madani TA, Ntoumi F, Kock R, Dar O, Ippolito G, McHugh TD, Memish ZA, Drosten C et al: The continuing 2019-nCoV epidemic threat of novel coronaviruses to global health - The latest 2019 novel coronavirus outbreak in Wuhan, China. Int J Infect Dis 2020, 91:264-266.

3. Paules $\mathrm{Cl}$, Marston HD, Fauci AS: Coronavirus Infections-More Than Just the Common Cold. JAMA 2020, 323(8):707-708.

4. Gorbalenya AE, Baker SC, Baric RS, de Groot RJ, Drosten C, Gulyaeva AA, Haagmans BL, Lauber C, Leontovich AM, Neuman BW et al: Severe acute respiratory syndrome-related coronavirus: The species and its viruses - a statement of the Coronavirus Study Group. bioRxiv 2020:2020.2002.2007.937862.

5. Statement on the second meeting of the International Health Regulations (2005) Emergency Committee regarding the outbreak of novel coronavirus (2019-nCoV) [https://www.who.int/newsroom/detail/30-01-2020-statement-on-the-second-meeting-of-the-internatio nal-health-regulations(2005)-emergency-committee-regarding-the-outbreak-of-novel-coronavirus-(201 9-ncov)]

6. WHO: Coronavirus disease 2019 (COVID-19): situation report, 45. In. Geneva: World Health Organization;

7. Zhu N, Zhang D, Wang W, Li X, Yang B, Song J, Zhao X, Huang B, Shi W, Lu R et al: A Novel Coronavirus from Patients with Pneumonia in China, 2019. N Engl J Med 2020, 382(8):727-733.

8. Chen N, Zhou M, Dong X, Qu J, Gong F, Han Y, Qiu Y, Wang J, Liu Y, Wei Y et al: Epidemiological and clinical characteristics of 99 cases of 2019 novel coronavirus pneumonia in Wuhan, China: a descriptive study. The Lancet 2020, 395(10223):507-513.

9. Huang C, Wang Y, Li X, Ren L, Zhao J, Hu Y, Zhang L, Fan G, Xu J, Gu X et al: Clinical features of patients infected with 2019 novel coronavirus in Wuhan, China. The Lancet 2020, 395(10223):497506.

10. WHO: Surveillance case definitions for human infection with novel coronavirus (nCoV). In.;

11. Diagnosis and Treatment of New Coronavirus Pneumonia (Sixth Edition) [http://www.nhc.gov.cn/yzygj/s7653p/202002/8334a8326dd94d329df351d7da8aefc2.shtml]

12. Laboratory biosafety guidance related to coronavirus disease 2019 (COVID19 [https://apps.who.int/iris/bitstream/handle/10665/331138/WHO-WPE-GIH-2020.1-eng.pdf]

13. Su S, Wong G, Shi W, Liu J, Lai ACK, Zhou J, Liu W, Bi Y, Gao GF: Epidemiology, Genetic Recombination, and Pathogenesis of Coronaviruses. Trends in Microbiology 2016:S0966842X16000718.

14. Wang D, Hu B, Hu C, Zhu F, Liu X, Zhang J, Wang B, Xiang H, Cheng Z, Xiong Y et al: Clinical Characteristics of 138 Hospitalized Patients With 2019 Novel Coronavirus-Infected Pneumonia in Wuhan, China. JAMA

15. Li Q, Guan X, Wu P, Wang X, Zhou L, Tong Y, Ren R, Leung KSM, Lau EHY, Wong JY et al: Early Transmission Dynamics in Wuhan, China, of Novel Coronavirus-Infected Pneumonia. New England 


\section{Journal of Medicine}

16. Wang C, Horby PW, Hayden FG, Gao GF: A novel coronavirus outbreak of global health concern. The Lancet 2020, 395(10223):470-473.

17. Zhou P, Yang XL, Wang XG, Hu B, Zhang L, Zhang W, Si HR, Zhu Y, Li B, Huang C-L et al: Discovery of a novel coronavirus associated with the recent pneumonia outbreak in humans and its potential bat origin; 2020.

18. Enserink, Science MJ: War Stories. 2013, 339(6125):1264-1268.

19. Yang X, Yu Y, Xu J, Shu H, Xia Ja, Liu H, Wu Y, Zhang L, Yu Z, Fang M et al: Clinical course and outcomes of critically ill patients with SARS-CoV-2 pneumonia in Wuhan, China: a single-centered, retrospective, observational study. The Lancet Respiratory Medicine

20. Xu X, Wu X, Jiang X, Xu K, Ying L, Ma C, Li S, Wang H: Clinical findings in a group of patients infected with the 2019 novel coronavirus (SARS-Cov-2) outside of Wuhan, China: retrospective case series. BMJ 2020, 368:m792.

21. De Wit E, van Doremalen N, Falzarano D, Munster VJJNRM: SARS and MERS: recent insights into emerging coronaviruses. 2016, 14(8):523-534.

22. Chen H, Guo J, Wang C, Luo F, Yu X, Zhang W, Li J, Zhao D, Xu D, Gong Q et al: Clinical characteristics and intrauterine vertical transmission potential of COVID-19 infection in nine pregnant women: a retrospective review of medical records. The Lancet 2020, 395(10226):809-815. 